\title{
The seismological and geodetical roles of strain seismogram suggested from the 2004 off the Kii peninsula earthquakes
}

\author{
Makoto Okubo, Yasuhiro Asai, Harumi Aoki, and Hiroshi Ishii \\ Tono Research Institute of Earthquake Science, Association for the development of Earthquake Prediction, \\ 1-63 Yamanouchi, Akeyo-cho, Mizunami, Gifu 509-6132, Japan
}

(Received November 30, 2004; Revised March 25, 2005; Accepted April 8, 2005)

\begin{abstract}
Dynamic strain seismograms obtained by high-speed sampling include not only long-period changes, such as 'strain-step', step change in strain, but also short period variations, such as body waves. We studied dynamic strain variations caused by the 2004 off the Kii peninsula earthquakes. Using such strain seismograms, we clarified the relation between dynamic strain and velocity. Our results will contribute greatly to seismology. We also clarified the strain-step formation process. This result and the concept of dynamic strain will bring more information to geodetics.
\end{abstract}

Key words: Dynamic strain seismogram, broadband velocity seismogram, global/local structure, strain-ramp function, geodetical strain-step.

\section{Introduction}

Large earthquakes with JMA (Japan Meteorological Agency) magnitude $\left(M_{\mathrm{JMA}}\right)$ of 7.1 and 7.4 occurred offshore southeast of the Kii Peninsula, Japan, on 5 September 2004. The origin time and hypocenter parameters determined by the JMA were as follows:

the foreshock (Eq. 1) :

$$
\begin{aligned}
& M_{\mathrm{JMA}}=7.1,19: 07: 07.50(10: 07: 07.50 \mathrm{UTC}), \\
& \mathrm{N} 33.030^{\circ}, \mathrm{E} 136.800^{\circ}, \text { depth }=38 \mathrm{~km} \\
& \text { the mainshock (Eq. } 2): \\
& M_{\mathrm{JMA}}=7.4,23: 57: 16.93(14: 57: 16.93 \mathrm{UTC}), \\
& \mathrm{N} 33.144^{\circ}, \mathrm{E} 137.142^{\circ}, \text { depth }=44 \mathrm{~km}
\end{aligned}
$$

Dynamic strain variations were observed by a borehole strain meter array located in Tono, central Japan (Fig. 1). These borehole instruments have multi-channel linear strain sensors which have a high-sensitivity response, and have been developed from the Ishii-type strain meter (Ishii et al., 2002). They incorporate other sensors including seismographs, tilt meters, magneto meters, and temperature sensors. Thus, we can comprehensively observe seismological, and geodetical events. Because a strain meter and a seismograph are installed in the same instrument, we can simultaneously obtain a dynamic strain seismogram and a broadband velocity seismogram at the same point. Thus, we can easily compare dynamic strain seismograms and broadband velocity seismograms.

\section{Data}

A borehole strain meter array consists of BYB, TGR350 and TGR165; instruments are installed at $-1020 \mathrm{~m},-350$ $\mathrm{m}$, and $-165 \mathrm{~m}$, respectively. TGR350 and TGR165 are located within $10 \mathrm{~m}$ horizontally of each other. Dynamic

Copy right(c) The Society of Geomagnetism and Earth, Planetary and Space Sciences (SGEPSS); The Seismological Society of Japan; The Volcanological Society of Japan; The Geodetic Society of Japan; The Japanese Society for Planetary Sciences; TERRAPUB. strain seismograms were recorded with $1 \mathrm{~Hz}$ sampling in TGR165 and TGR350, and with $20 \mathrm{~Hz}$ sampling in BYB. In this study, we focus on dynamic strain variations among the horizontal plane, and treat the vertical component where possible. Borehole instrument TGR350 is not equipped vertical component sensor. Dynamic strain variations which observed at BYB, TGR165 and TGR350 are shown in Fig. 2. To display the multi-channel dynamic strain seismogram, we use the diagram of Okubo et al. (2004). This is useful to indicate variations in dynamic strain which have been caused by azimuthal change. In the diagram, time varies toward the radial direction along the installation direction. The amplitude varies counter-clockwise with extensional strain, and clockwise with compressional strain.

We also used seismograms derived from instrument TGS165 and N.NAAF. TGS165 is broadband velocity seismograph installed with the instruments in TGR165. N.NAAF is an vault-type F-net observatory operated by the National Research Institute for Earth Science and Disaster Prevention. Broadband seismogram of TGS165 and N.NAAF were recorded with $100 \mathrm{~Hz}$ sampling.

Because the 2004 off the Kii peninsula earthquakes occurred near the observatories, the amplitudes of the velocity seismograms of N.NAAF and TGS165 were a little saturated for large-amplitude variations, such as Rayleigh waves. But the dynamic strain seismogram was not saturated for the same waves.

\section{Analysis}

'Strain-steps' which are geodetical phenomenon can be identified clearly even from those diagrams shown in Fig. 2. It appears that the dynamic strain seismograms have same radiation patterns as broadband velocity seismograms. It seems that the amplitude of dynamic strain variation is large along the radial direction shown by the solid arrow and small along the transverse direction. It further seems that 


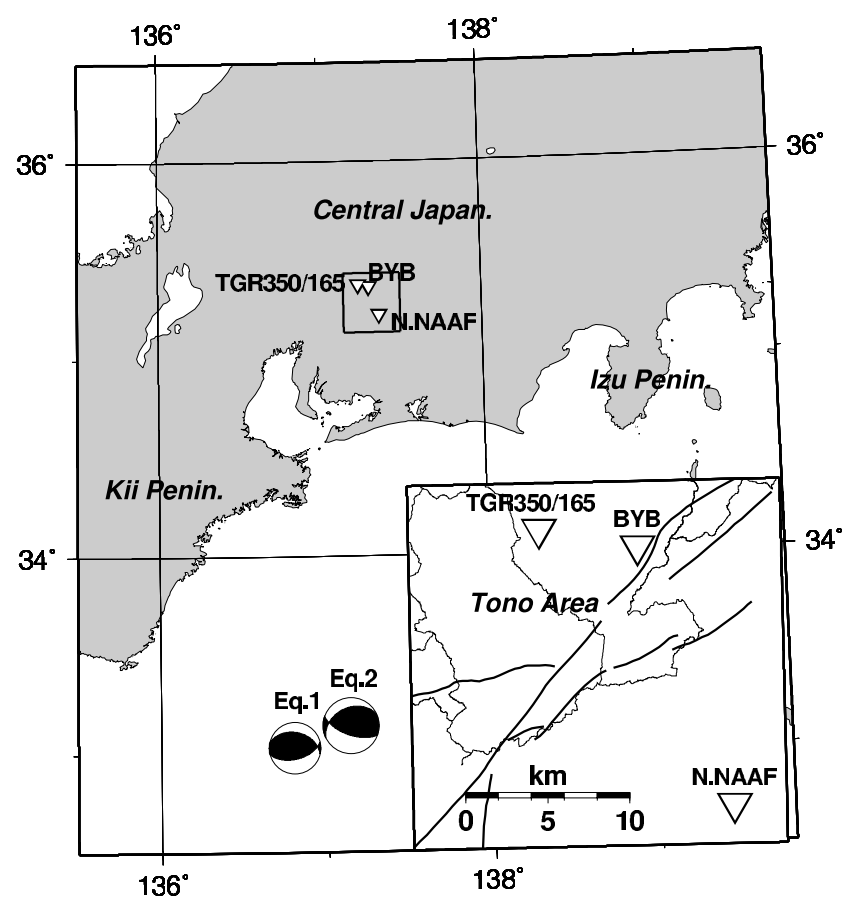

Fig. 1. Location of crustal activity observatories and hypocenters of the 2004 off the Kii peninsula earthquakes. BYB is the deepest borehole-type station for observing multi-component crustal activity; instruments are installed at $-1020 \mathrm{~m}$ from the ground. TGR350 $(-350 \mathrm{~m})$ and TGR165 $(-165 \mathrm{~m})$ are also borehole observatories, and are located within $10 \mathrm{~m}$ horizontally of each other. N.NAAF is a vault-type broadband velocity seismograph observatory with $97 \mathrm{~m}$ length operated by the National Research Institute for Earth Science and Disaster Prevention.

the dynamic strain variation is similar to the seismograph variation from the waveform comparison results. Our observation prompted some questions:

1) Does the strain meter for long-term observations respond correctly up to the same frequency range as a seismograph? And what relation exists between their observations?

2) Can the 'strain-step' that was seen only by geodetical observations be observed by seismological analysis? How and when does it form?

We performed two analyses in order to answer these questions. One was a survey to identify the seismological role of dynamic strain seismograms. The other was an analysis to clarify the geodetical features of a dynamic strainstep.

\subsection{Analysis of seismological role}

Because the 2004 off the Kii peninsula earthquakes occurred near the observation, the dynamic strain seismogram and broadband velocity seismogram included higherfrequency waves, so we compared the seismograms at a short period range by using body waves. This analysis can clarify the instrumental response of the strain meter and the relation of the shear contributions between dynamic strain seismograms and velocity seismograms from the long period to the short period. In our previous study (Okubo et al., 2004), we compared a strain seismogram with a broadband velocity seismogram of surface waves with a frequency of less than $1 \mathrm{~Hz}$ for the 2003 Tokachi oki Earthquake. The strain seismogram had the same features as those of the broadband velocity seismogram about the surface waves of large distance. We obtained the following relation between strain seismogram $\left(\varepsilon_{x x}, \varepsilon_{y y}\right)$ with velocity seismogram $\left(S_{x}\right.$, $\left.S_{y}\right)$ :

$$
S_{x, y}=\alpha \cdot \varepsilon_{x x, y y},
$$

where

$$
\alpha=5000(\mathrm{~m} / \mathrm{s} / \mathrm{strain})
$$

Because the observatories that collected our previous data are far from the hypocenter, we compared the seismograms only over the long period. Although the direction we used for that analysis might not be consistent with the principal axes, the relation does not include a shear contribution.

To compare dynamic strain seismograms with broadband velocity seismograms, datasets with the same directional component are required. So we calculated the radial $\left(\varepsilon_{r r}\right)$, transverse $\left(\varepsilon_{t t}\right)$, and shear $\left(\varepsilon_{r t}, \varepsilon_{t r}\right)$ components of the strain seismograms by using strain tensor analysis. We decided on the radial direction to make shear contribution easy to take into consideration. The shear component works in the normal plane of the radial direction $\left(\varepsilon_{r t}\right)$ and transversely $\left(\varepsilon_{t r}\right)$, and its amplitudes are the same in analysis. The broadband velocity seismogram was directed toward the epicenter by rotational analysis, then radial $\left(S_{r}\right)$ and transverse components $\left(S_{t}\right)$ of the seismograms were calculated.

From the expression of strain in Okada (1981), the directions of principal strain were obtained from the following equations:

$$
\begin{aligned}
\tan \theta_{1,2} & =\frac{e_{1,2}-\varepsilon_{r r}}{\varepsilon_{r t}} \\
& =\frac{\varepsilon_{r t}}{e_{1,2}-\varepsilon_{t t}}
\end{aligned}
$$

where

$$
\theta_{2}=\theta_{1} \pm \frac{\pi}{2}
$$

$\theta$ is the angle between the principal strain axis and the observation axis $\left(\varepsilon_{r r}\right)$. From these equations, we can obtain the relationship between principal strain and observations:

$$
\begin{aligned}
e_{1} & =\varepsilon_{r r}+\varepsilon_{r t} \tan \theta_{1}, \\
e_{2} & =\varepsilon_{t t}+\varepsilon_{r t} \frac{1}{\tan \theta_{2}}, \\
& =\varepsilon_{t t}-\varepsilon_{r t} \tan \theta_{1} .
\end{aligned}
$$

Here, the range which an angle $\theta_{1}$ can take is as follows:

$$
\frac{\pi}{4} \leq \theta_{1} \leq \frac{\pi}{4}
$$

In seismological analysis, the component has maximum amplitude when the observational axis is directed toward the hypocenter. That is, the second term of Eqs. (1) and (2) (the shear contribution) is zero. Thus, the principal strain axis and radial direction are in agreement. When the axis is inharmonious, we think that the local structure, which 


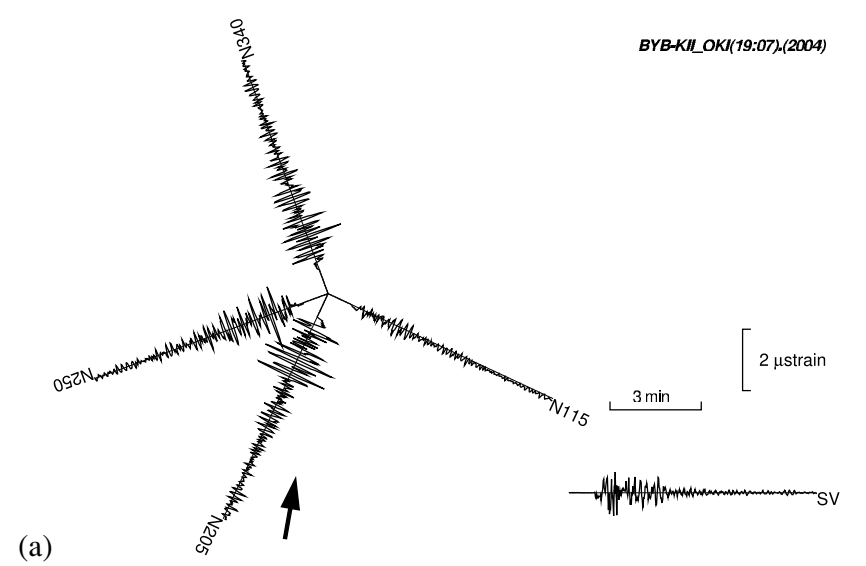

(a)

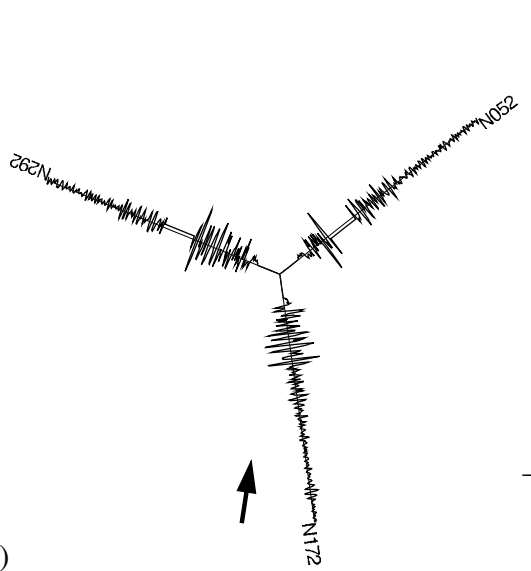

TGR165-KII_OKL.(19:07).(2004)

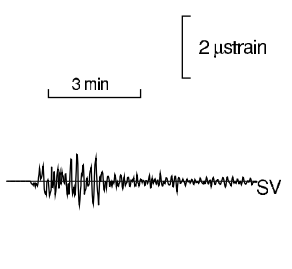

TGR350-KILOKL,(19:07).(2004)

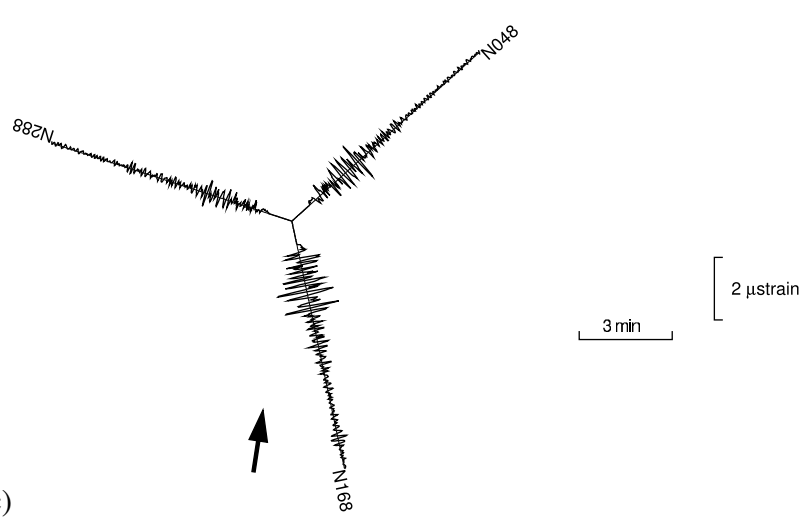

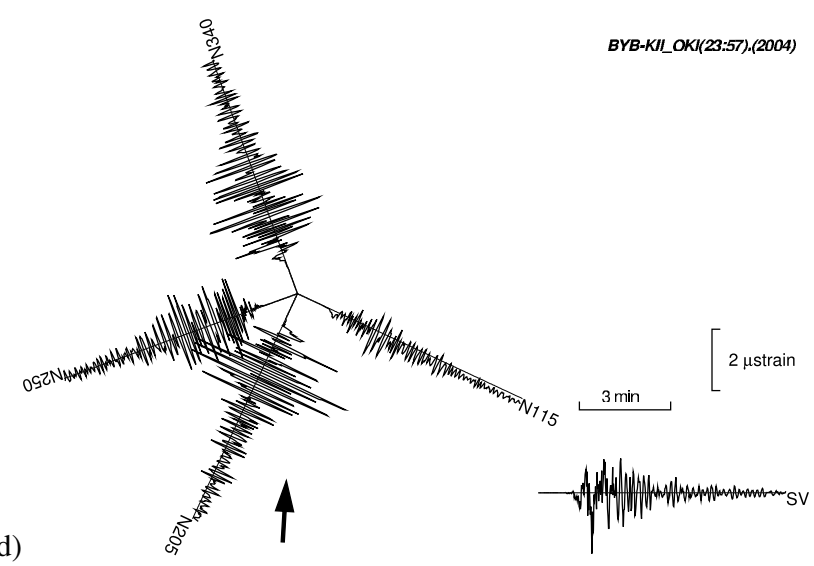

TGR165-KII_OKL.(23:57).(2004)
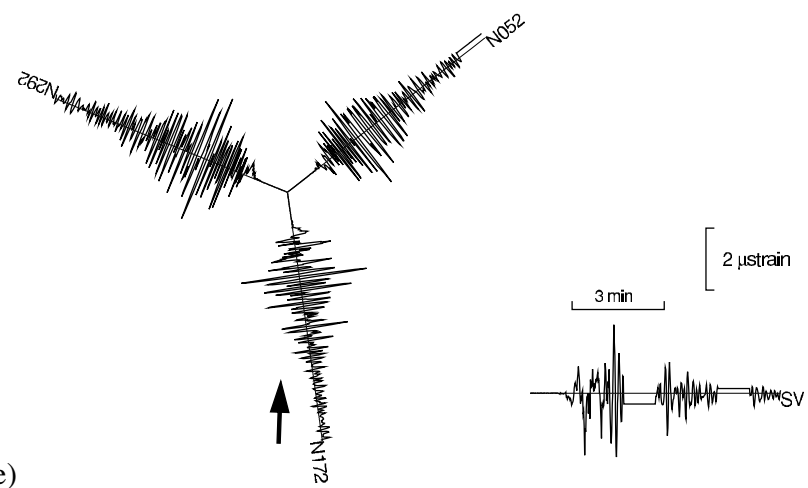

TGR350-KII_OKI.(23:57).(2004)

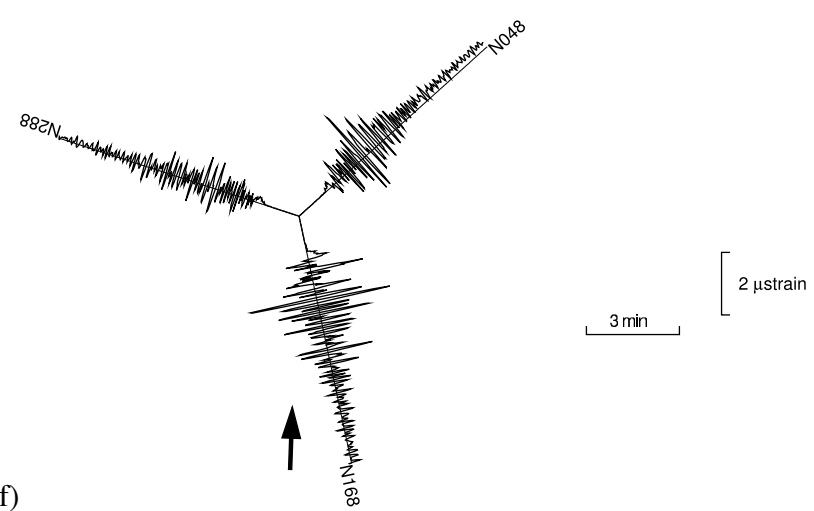

Fig. 2. Dynamic strain seismograms from BYB, TGR165 and TGR350. The coordinate origin is the origin time of the earthquake. The amplitude of compressional strain varied toward clockwise; that of extensional strain varied counter-clockwise. Since BYB and TGR165 also observed vertical component strain, these are shown with the other components. Solid arrow indicates wave propagation from the epicenter. Strain seismograms (a), (b) and (c) are plotted for the foreshock (Eq. 1), and (d), (e) and (f) are plotted for the mainshock (Eq. 2). The TGR165 records are incomplete, owing to data loss.

is related to the medium anisotropy near an observation station, disturbs the direction of principal strain.

We made compared dynamic strain seismograms with velocity seismograms with and without shear contribution. The results are shown in Figs. 3 and 4. The validity of the comparison is evaluated with a cross-correlation factor. The result of correlation analysis of waveform comparison shown in Table 1 indicates that we must consider the effect of anisotropic media when dealing with dynamic strain seismograms and velocity seismograms. But the shear contribution to the waveform can be disregarded when we con- sider only long-period waves such as in Okubo et al. (2004), in which wave-length is larger than local structure.

We also compared spectra between dynamic strain seismograms and velocities to obtain the ratio of amplitude as reported by Mikumo and Aki (1964) and Sacks et al. (1976). The result for the transverse component, which includes shear contribution, is shown in Fig. 5. The ratio of amplitude varies between 2000 and 5000, and it depends on frequency, so it seems that the ratio is influenced by structure, such as the existence of inhomogeneous media. Otherwise, transfer velocity might depend on $P$-wave velocity for 
(a)
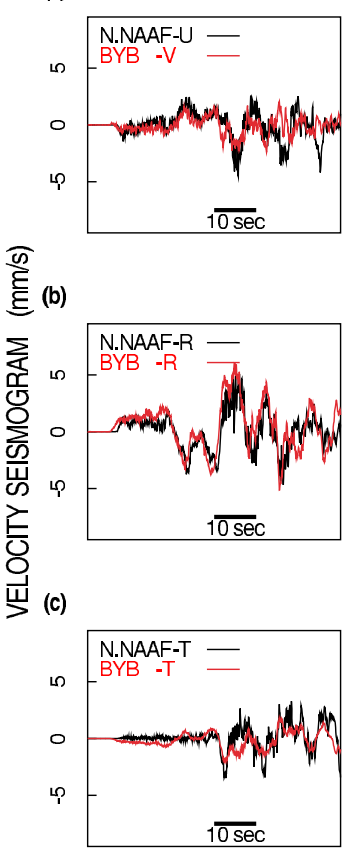

(d)

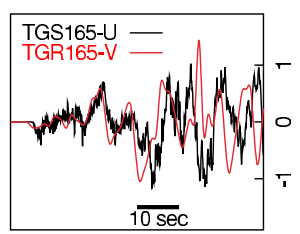

(e)

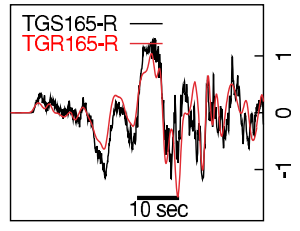

(f)

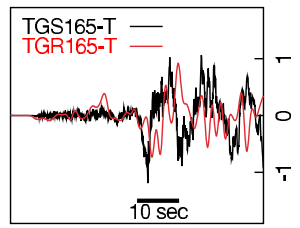

Fig. 3. Waveform comparison between dynamic strain seismogram and broadband velocity seismogram. The dynamic strain seismogram does not include the effect of shear component. (a)-(c) TGR165 vs. TGS165; (d)-(f) BYB vs. N.NAAF. (a) and (d), Vertical components; (b) and (e), radial component; (c) and (f), transverse.

(a)

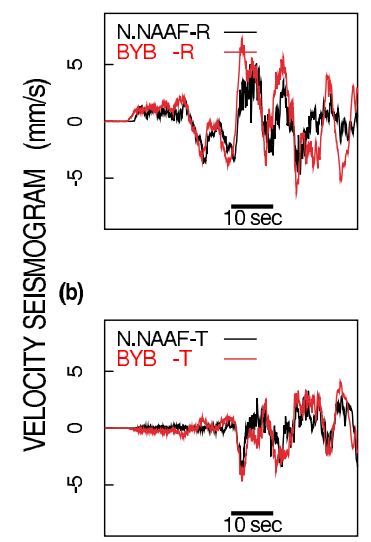

(c)

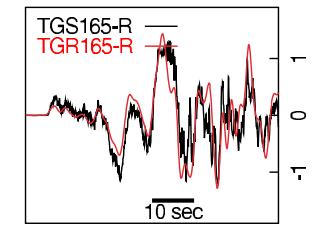

(d)

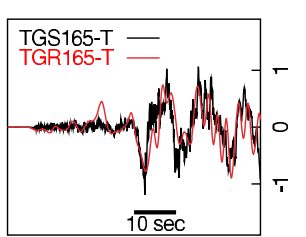

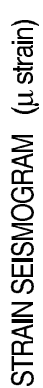

Fig. 5. Spectral comparison between dynamic strain and velocity seismogram. spectrum is calculated for the transverse component, which includes shear contribution in the case of strain. The ratio of amplitude between velocity and strain is shown (right hand axis).

Table 1. Cross-correlation results of waveform comparison.

\begin{tabular}{ccc}
\hline & only $e_{1,2}$ & include $\varepsilon_{r t}$ \\
\hline TGS165 vs. & & \\
TGR165 & & \\
radial & -0.63 & -0.67 \\
transverse & -0.40 & -0.86 \\
BYB & & \\
radial & -0.68 & -0.71 \\
transverse & -0.85 & -0.87 \\
N.NAAF vs. & & \\
TGR165 & & -0.57 \\
radial & -0.52 & -0.67 \\
transverse & -0.32 & \\
BYB & & -0.76 \\
radial & -0.65 & -0.87 \\
transverse & -0.82 & \\
\hline
\end{tabular}

waves, $M=\mu$. In the case of large distances as reported by Okubo et al. (2004), the ratio is 5000 on average. This result thought as a $P$-wave velocity and our present result thought as a $P$-, and $S$-wave velocity are satisfied when: $\lambda=17.5 \mathrm{GPa}, \mu=22.5 \mathrm{GPa}$, and $\rho=2500 \mathrm{~kg} / \mathrm{m}^{3}$.

Thus, we conclude that we must consider the effects of inhomogeneous media when we deal with dynamic strain seismograms and velocity seismograms. The relation between a strain seismogram and a velocity seismogram is as follows:

$$
S_{r, t}=\sqrt{M / \rho} \cdot\left(\varepsilon_{r r, t t} \pm \varepsilon_{r t} \tan \theta\right) .
$$

From this analysis of seismological role, we clarified that our borehole-type strain meter has very broadband response, and suggest that dynamic strain seismograms can (4) be used with broadband velocity seismograms, not only for long-period waves, but also short-period waves, such as body waves. Thus analyses which use dynamic strain

where $M$ is modulus. For $P$-waves, $M=\lambda+2 \mu$; for $S$ - 
Table 2. Quantities of strain-step, observed at BYB, TGR165, and TGR350 after $300 \mathrm{~s}$.

\begin{tabular}{|c|c|c|c|c|c|c|c|}
\hline & \multicolumn{5}{|c|}{ This study } & \multicolumn{2}{|c|}{ Asai et al. } \\
\hline & $\varepsilon_{r r}$ & $\varepsilon_{r t}$ & $\varepsilon_{t t}$ & $\varepsilon_{\max }$ & $\varepsilon_{\min }$ & $\varepsilon_{\max }$ & $\varepsilon_{\min }$ \\
\hline \multicolumn{8}{|l|}{ Eq. (1) } \\
\hline BYB* & 9.8 & -5.1 & -12.5 & $\simeq 10.9$ & $\simeq-13.6$ & 11.2 & -15.1 \\
\hline TGR350 & 8.1 & 2.3 & -3.5 & $\simeq 8.5$ & $\simeq-3.9$ & 8.8 & -4.2 \\
\hline TGR165 & 3.3 & -1.0 & -2.2 & $\simeq 3.4$ & $\simeq-2.4$ & 3.4 & -3.4 \\
\hline \multicolumn{8}{|l|}{ Eq. (2) } \\
\hline BYB* & 28.1 & -11.6 & -33.7 & $\simeq 30.2$ & $\simeq-35.8$ & 29.6 & -37.7 \\
\hline TGR350 & 31.1 & 28.4 & 3.8 & $\simeq 49.0$ & $\simeq-14.0$ & 51.7 & -16.7 \\
\hline TGR165 & 9.8 & -1.6 & -6.6 & $\simeq 9.9$ & $\simeq-6.8$ & 11.1 & -8.6 \\
\hline
\end{tabular}

The respective solution was calculated with N115, N205, and N340 components

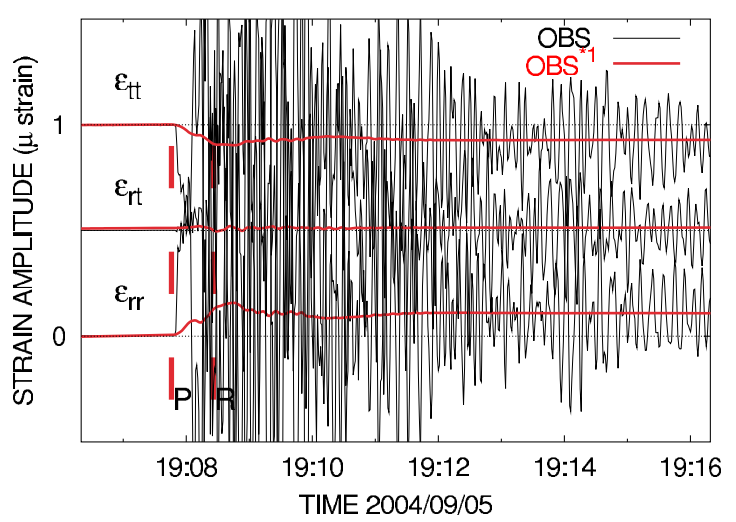

Fig. 6. Strain-ramp function obtained by the application of a low-pass filter. Strain components $\varepsilon_{r r}, \varepsilon_{r t}$ and $\varepsilon_{r r}$ are shown. $P$ - and Rayleigh-wave arrival times are indicated; they were calculated theoretically for $V_{p}=6.9 \mathrm{~km} / \mathrm{s}$ and $V_{r}=3.2 \mathrm{~km} / \mathrm{s}$.

seismograms and velocity seismograms simultaneously are useful for detecting local and/or global structure.

\subsection{Analysis of geodetical role}

To clarify the geodetical role of dynamic strain seismograms, we must obtain very long-period changes by removing dynamic variations from observation records. We applied a low-pass filter to the records and obtained the geodetical strain-step shown in Fig. 6. The filter parameters were stop-band frequency $\left(f_{s}\right) 50 \mathrm{mHz}(=0.05 \mathrm{~Hz})$ and corner frequency $\left(f_{c}\right) 1 \mathrm{mHz}$. The zero level shifted not instantaneously but gradually. Thus the function is a strain-ramp function, not a strain-step function. Although various type of filters were applied, the long-term nature and the quantity of the final step did not change (Fig. 7). The strain-ramp function changes its amplitude for large-amplitude waves such as $P$-, and Rayleigh waves, and fixes its amplitude when they pass.

In past studies, there was an assumption that a strainstep was formed over a much shorter time than the duration of geodetical events. Since the data sampling intervals in past geodetical observations were very large, it may have seemed that the strain shifted the zero level in an instant. Wideman and Major (1967) reported that the transfer ve-

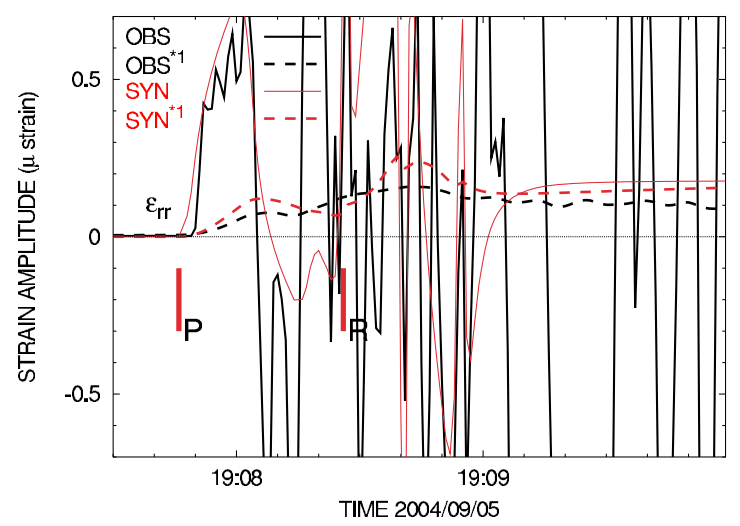

Fig. 7. Suitability of strain-ramp function obtained by filtering. Black line: observation waveforms; read line: synthetic waveforms; dashed line: filtered records. $P$ - and Rayleigh-wave arrival times are indicated; they were calculated theoretically for $V_{p}=6.9 \mathrm{~km} / \mathrm{s}$ and $V_{r}=3.2$ $\mathrm{km} / \mathrm{s}$.

locity of a strain-step is $3.0 \mathrm{~km} / \mathrm{s}$ for continental crust and $3.6 \mathrm{~km} / \mathrm{s}$ for oceanic crust. The transfer velocities may be equivalent to the passage time of the maximum amplitude wave in our analysis. Okada (1980) reported the result of a theoretical analysis of the strain-step formation process. In his theory, it starts to form a strain-step by $P$-wave arrival and continues formation in the later phase. The feature of our observation shown in Figs. 6 and 7 support this theory of the strain-step formation process.

The final quantities of geodetical strain-steps estimated from the filtered records are summarized in Table 2. BYB has four strain tensor analysis solutions. The respective solution of BYB was calculated with N115, N205, and N340 components. These results are well consistent with the results of Asai et al. (2005). Because their study and ours used the same dataset, this result is to be expected. They analyzed long-term variation with tidal analysis, such as weekly. But, we analyzed dynamic strain seismograms only 300 s. Thus, strain-step quantities in geodetical meaning can be estimated by using seismological techniques, such as the application of a low-pass filter to records. By using a dynamic strain seismogram, we also clarified the process of 
Table 3. Quantities of strain-step, predicted by Okada's (1980) calculation.

\begin{tabular}{cccc}
\hline & $\varepsilon_{r r}$ & $\varepsilon_{r t}$ & $\varepsilon_{t t}$ \\
\hline Eq.1 & 9.3 & -1.6 & -3.9 \\
Eq.2 & 24.5 & 2.7 & -9.7 \\
\hline & & \multicolumn{3}{r}{$\times 10^{-8}$ strain }
\end{tabular}

formation of a strain-ramp function, which is a strain-step in traditional usage.

\section{Discussion and Conclusions}

From the analysis of seismological role, we suggest that dynamic strain seismograms can be used with broadband velocity seismograms, not only for long-period waves but also for short-period waves such as body waves.

Thus, we obtained the new instruments, such as boreholetype strain meter which has quite wide-band response, for broadband seismological analysis.

We clarified the relation between dynamic strain seismograms and broadband velocity seismograms; it indicated the need to consider the structure. Analysis using dynamic strain seismograms and velocity seismograms simultaneously is a useful method to detect local and/or global structure.

By using a dynamic strain seismogram, we also clarified the process of formation of the strain-ramp function, which is a strain-step by traditional usage. The zero level of a dynamic strain seismogram shifted not instantaneously but gradually. The strain-ramp function changes its amplitude for large-amplitude waves such as $P$-, $S$-, and Rayleigh waves and finally fixes its amplitude when they pass. Thus, strain-step quantities in geodetical meaning can be estimated by using seismological techniques.

The quantity of a strain-step in geodetical meaning is summarized in Table 3. Those results are calculated with: $\mu=20 \mathrm{GPa}, V_{p}=6.9 \mathrm{~km} / \mathrm{s}$, and earthquake mechanism parameters (Park and Mori, 2005). Our result is not consistent with the theoretical calculations summarized in Table 3, except for the component $\varepsilon_{r r}$. This shows that the assumption of the half-infinity homogeneous medium by Okada (1980) is not materialized any longer. To analyze dynamic variation in inhomogeneous medium, it seems necessary to build new models.
Thus, high sampled dynamic strain seismograms provide more seismological and geodetical information (cf. Koyama et al., 2004, high sampled GPS data). The dynamic strain seismogram can play an important role in seismological short-period phenomena by geodetical observations and in clarifying geodetical long-period phenomena by using seismological methods.

Acknowledgments. The broadband velocity seismograph data (N.NAAF) used in the present study were provided by the National Research Institute for Earth Science and Disaster Prevention. We were grateful to Alan Linde and Tsuneo Yamauchi for providing useful comments.

\section{References}

Aki, K. and P. G. Richards, Quantitative Seismology, W. H. Freeman and Company, San Francisco, 932 pp., Second Edition, 2002, University Science Books, Sansalito, California, 699 pp., 1980.

Asai, Y., M. Okubo, H. Ishii, H. Aoki, T. Yamauchi, Y. Kitagawa, N. Koizumi, and S. Azuma, Co-seismic strain-steps associated with the 2004 off the Kii peninsula earthquakes-Observed with Ishii-type borehole strainmeters and quartz-tube extensometers, Earth Planets Space, 57, this issue, 309-314, 2005.

Ishii, H., T. Yamauchi, S. Matsumoto, Y. Hirata, and S. Nakano, Seismogenic Process Monitoring, edited by H. Ogasawara, T. Yanagitani, and M. Ando, A. A. Balkema, Tokyo, 414 pp, 2002.

Japan Meteorological Agency, Monthly Report on Earthquakes and Volcanoes in Japan October 2004, edited by Japan Meteorological Agency, 186, 2004.

Koyama, J., N. V. Shestakov, and R. Honda, Strong ground motion recorded by High-rate sampling GPS at the closest site to the 2003 Tokachi-oki earthquake, Earth Planets Space, 56, 383-387, 2004.

Mikumo, T. and K. Aki, Determination of local phase velocity by intercomparison of seismograms from strain and pendulum instruments, $J$. Geophys. Res., 69, 721-731, 1964.

Okada, Y., Theoretical strain seismogram and its applications, Bull. Earthq. Res. Inst. Tokyo Univ., 55, 101-168, 1980 (in Japanese with English abstract).

Okada, Y., Confusions on the expressions in strain analysis, J. Seismol. Soc. Jpn 2, 34, 148-152, 1981 (in Japanese).

Okubo, M., H. Ishii, and T. Yamauchi, The 2003 Tokachi-oki earthquake, observed by borehole strainmeter array-Comparison with broadband seismogram, J. Seismol. Soc. Jpn 2, 57, 105-114, 2004 (in Japanese with English abstract).

Park, S-C. and J. Mori, The 2004 sequence of triggered earthquakes off the Kii peninsula, Japan, Earth, Planets Space, 57, this issue, 315-320, 2005

Sacks, I. S., J. A. Snoke, R. E. G. King, and J. Beavan, Geophys. J. R. astr. Soc., 46, 253-258, 1976.

Wideman, C. J. and M. W. Major, Strain steps associated with earthquakes, Bull. Seism. Soc. Am., 57, 1429-1444, 1967.

M. Okubo (e-mail: okubo@tries.jp), Y. Asai, H. Aoki, and H. Ishii 